Check for updates

Cite this: RSC Adv., 2018, 8, 36812

Received 5th June 2018

Accepted 17th October 2018

DOI: $10.1039 / \mathrm{c} 8 \mathrm{ra} 04792 \mathrm{~h}$

rsc.li/rsc-advances

\title{
Twisted molecule-based hyper-crosslinked porous polymers for rapid and efficient removal of organic micropollutants from water $\uparrow$
}

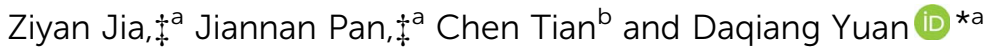

Organic micropollutants have become serious threats to human health and the environment over the past years. Novel materials and technologies are urgently needed to remove environmental contaminants. Herein, spirobifluorene (SBF) and triptycene (TP) were crosslinked to produce four hierarchically porous organic polymers (NHCPs). The twisted spatial structures of the monomers endow the NHCPs with high surface areas and abundant pores, which make them suitable for removal of pollutants in aqueous solution through adsorption. According to the results from adsorption experiments, the NHCPs exhibited high removal efficiency and adsorption capacities for pollutants in solution. Particularly, NHCP-3 could remove $99.4 \%$ of bisphenol $A$ (BPA) in a few seconds with a maximum adsorption capacity of $562 \mathrm{mg}$ $\mathrm{g}^{-1}$. Furthermore, the NHCPs could be easily recovered by immersion in ethanol and recycled at least five times without a loss in efficiency.
\end{abstract}

\section{Introduction}

Organic micropollutants in water, including natural organic matter (NOM) and synthetic organic compounds (SOCs), are generally regarded as enormous threats to human health and the environment. ${ }^{1-3}$ NOMs are usually produced by the decomposition of plants and animals, which are precursors of most disinfection by-products. They form various halogenated organic compounds after chlorination of water. SOCs primarily comprise toxic and harmful substances, such as pesticides, pharmaceuticals and chemical products, which are emitted in agricultural and industrial wastewater. Most of them are endocrine disruptors with carcinogenicity, neurotoxicity and reproductive toxicity, which directly harm human health. ${ }^{4-6}$ In particular, SOCs have become more abundant in species and quantity with the rapid industrial development in recent years. Currently, the micropollutant treatment technologies being applied mainly involve adsorption, chemical oxidation, photocatalytic degradation, and membrane filtration. ${ }^{-11}$ Among them, physical adsorption is an efficient and low-cost treatment method based on the interaction force between adsorbents and adsorbates. The adsorption efficiency depends on multiple

${ }^{a}$ Fujian Institute of Research on the Structure of Matter, Chinese Academy of Sciences, Fuzhou, Fujian 350002, China. E-mail: ydq@fjirsm.ac.cn

${ }^{b}$ School of Environment and Energy, Key Laboratory of Pollution Control and Ecosystem Restoration in Industry Clusters, Ministry of Education, Guangdong Engineering and Technology Research Center for Environmental Nanomaterials, South China University of Technology, Guangzhou 510006, China

$\dagger$ Electronic supplementary information (ESI) available: Characterization data and properties. See DOI: 10.1039/c8ra04792h

\$ These authors contribute to this work equally. features of the adsorbents, including the porosity, the composition and surface properties. The development of more efficient adsorbing materials has attracted significant attention of researchers worldwide.

In order to satisfy the standards required for pollution cleanup, considerable efforts have been devoted to exploring novel porous materials with high adsorption efficiency and capacity for the treatment of contaminated water. At present, activated carbon (AC) is one of the most widely applied adsorbents owing to its high surface area, which endows it with excellent adsorption capacity for wastewater treatment and prevention of air pollution..$^{12-15}$ However, it cannot be ignored that the adsorption efficiency of ACs gradually decreases overtime after the saturation of active sites and the regeneration process requires large energy, with temperatures in excess of $500{ }^{\circ} \mathrm{C}$. Recently, in addition to ACs, metal-organic frameworks (MOFs) and porous organic polymers (POPs) have also undergone rapid development for use in pollutant removal. Featuring high porosity and tunable pore sizes, MOFs can be specially designed according to the characteristics of the targeted pollutants, which make them promising materials for the selective removal of hazardous compounds. ${ }^{16-20}$ Unfortunately, most MOFs are sensitive to acids or bases and sometimes suffer from hydrolysis. In contrast, POPs are highly stable in extreme environments and show tremendous potential for practical water decontamination because of their strong covalent bonding. ${ }^{21-26}$ As a typical example, Dichtel et al. reported a $\beta$ cyclodextrin-based porous polymer with an adsorption rate constant of up to 200 times larger than that of ACs, and realizes the rapid and efficient removal of pollutants. ${ }^{21}$ However, the preparation of POPs usually requires high temperature, long 


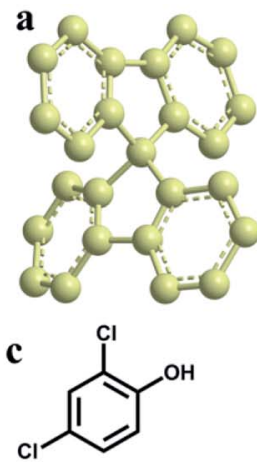

2,4-DCP<smiles>Oc1ccc2ccccc2c1</smiles>

2-NO b
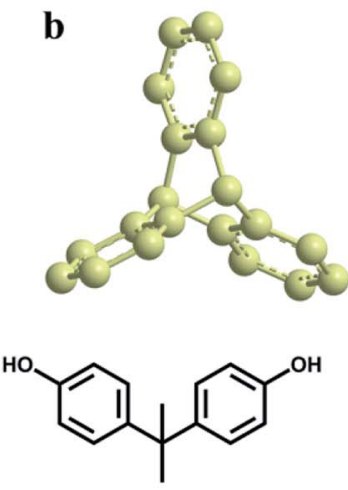

BPA

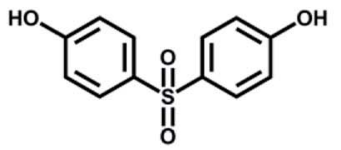

BPS
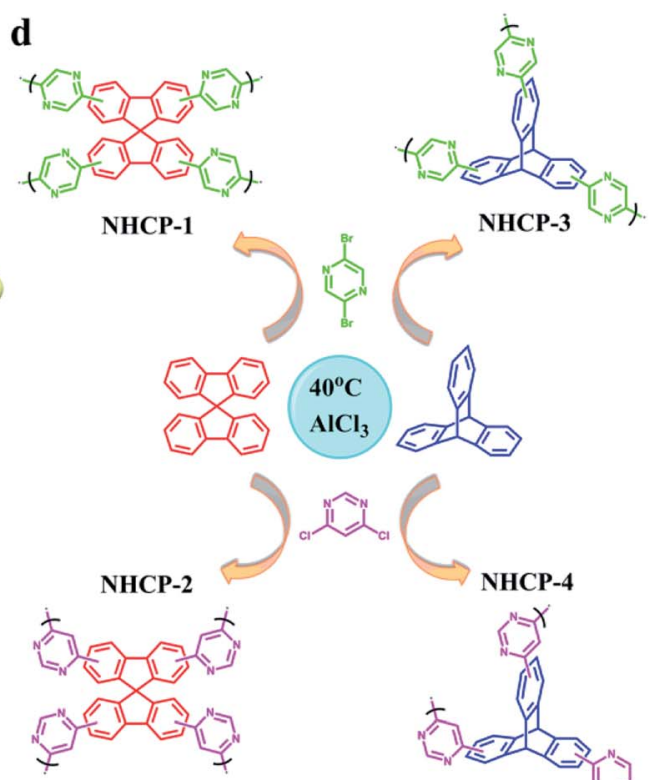

年
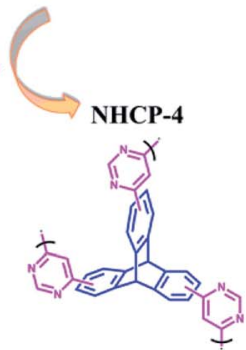

Fig. 1 (a) The spatial structure of SBF. (b) The spatial structure of TP. (c) Four organic micropollutants used in the experiment. (d) The synthetic routes for NHCPs.

reaction time and noble metal catalysts, which are unfavorable for large-scale industrial production. Hence, the facile preparation of materials becomes a primary concern.

Herein, we synthesized four N-containing hyper-crosslinked porous polymers, namely, NHCP-1, NHCP-2, NHCP-3 and NHCP-4 with a facile method based on our previous report. ${ }^{27}$ The polymerization occurred at a relatively low temperature (40 ${ }^{\circ} \mathrm{C}$ ) under the catalysis of inexpensive aluminium chloride. Two bulk molecules with twisted spatial structures (Fig. 1a and b), namely, spirobifluorene (SBF) and triptycene (TP), were adopted to be crosslinked by 2,5-dibromopyrazine (2,5-DBP) and 4,6dichloropyrimidine (4,6-DCM) to obtain porous organic polymers with large surface areas of up to $1768 \mathrm{~m}^{2} \mathrm{~g}^{-1}$. SBF and TP were selected as monomers to construct the hyper-crosslinked polymers due to their unique spatial structures and intrinsic microporosity. ${ }^{28}$ The twisted molecule crosslinked by rigid crosslinkers contributes to the construction of hierarchically porous polymers, which are suitable for the adsorption removal of bulky organic pollutants. 2,4-dichlorophenol (2,4-DCP), 2naphthol (2-NO), bisphenol A (BPA) and bisphenol S (BPS) were selected to be simulated pollutants from wastewater, as shown in Fig. 1c. The NHCPs exhibited high adsorption capacities towards the organic small molecules in aqueous solution and could remove them rapidly and efficiently through fast flow experiments. The excellent adsorption performance of the NHCPs benefits from their abundant porosity and high surface areas, which makes these NHCPs potential wastewater cleaning materials.

\section{Experiments}

\subsection{Materials experiments}

Spirobifluorene (SBF) (98\%), triptycene (TP) (98\%), 2,5-dibromopyrazine (2,5-DBP) (98\%) and 4,6-dichloropyrimidine (4,6-
DCM) (98\%) were purchased from Adamas. Aluminium chloride anhydrous (99\%) was purchased from Alfa. All other chemicals for the synthesis were obtained from commercial sources and used without further purification.

\subsection{Preparation of NHCP-1}

Dichloromethane $(30 \mathrm{~mL})$ was injected along with $\mathrm{SBF}$ (1.6 mmol, $0.51 \mathrm{~g}$ ), 2,5-DBP ( $3.2 \mathrm{mmol}, 0.76 \mathrm{~g}$ ) and anhydrous aluminium chloride ( $10 \mathrm{mmol}, 1.33 \mathrm{~g}$ ) in a $100 \mathrm{~mL}$ roundbottom flask under inert atmosphere. The mixture was stirred for $24 \mathrm{~h}$ at $40^{\circ} \mathrm{C}$ to complete the polymerization. After cooling to room temperature, the reaction was quenched with a small amount of methanol. The solution was filtered to collect the residue under extraction filtration. Then, the residue was washed with diluted hydrochloric acid, methanol and dichloromethane, in sequence. For further purification, methanol was used to extract the crude product with Soxhlet extractor for $24 \mathrm{~h}$. Yellow powder was obtained after drying in a vacuum oven at $120^{\circ} \mathrm{C}$ for $8 \mathrm{~h}$. Yield: $0.69 \mathrm{~g}$. Elemental anal. C: 82.41\%; H: 4.43\%; N: 1.81\%; Al: 0.021\% (the content of Al was measured by ICP analysis).

\subsection{Preparation of NHCP-2}

Dichloromethane $(30 \mathrm{~mL})$ was injected along with SBF ( $1.6 \mathrm{mmol}, 0.51 \mathrm{~g}), 4,6-\mathrm{DCM}(3.2 \mathrm{mmol}, 0.48 \mathrm{~g})$ and anhydrous aluminium chloride $(10 \mathrm{mmol}, 1.33 \mathrm{~g})$ in a $100 \mathrm{~mL}$ roundbottom flask under inert atmosphere. The mixture was stirred for $24 \mathrm{~h}$ at $40^{\circ} \mathrm{C}$ to complete the polymerization. After cooling to room temperature, the reaction was quenched with a small amount of methanol. The solution was filtered to collect the residue under extraction filtration. Then, the residue was washed with diluted hydrochloric acid, methanol and dichloromethane, in sequence. For further purification, 
methanol was used to extract the crude product with Soxhlet extractor for $24 \mathrm{~h}$. Brick-red powder was obtained after drying the product in a vacuum oven at $120{ }^{\circ} \mathrm{C}$ for $8 \mathrm{~h}$. Yield: $0.76 \mathrm{~g}$. Elemental anal. C: $78.89 \%$; H: 4.30\%; N: 4.91\%; Al: $0.031 \%$ (the content of $\mathrm{Al}$ was measured by ICP analysis).

\subsection{Preparation of NHCP-3}

Dichloromethane $(30 \mathrm{~mL})$ was injected along with TP (1.6 mmol, $0.41 \mathrm{~g}$ ), 2,5-DBP (2.4 mmol, $0.57 \mathrm{~g}$ ) and anhydrous aluminium chloride ( $10 \mathrm{mmol}, 1.33 \mathrm{~g}$ ) in a $100 \mathrm{~mL}$ roundbottom flask under inert atmosphere. The mixture was stirred for $24 \mathrm{~h}$ under $40{ }^{\circ} \mathrm{C}$ to complete the polymerization. After cooling to room temperature, the reaction was quenched with a small amount of methanol. The solution was filtered to collect the residue under extraction filtration. Then, the residue was washed with diluted hydrochloric acid, methanol and dichloromethane, in sequence. For further purification, methanol was used to extract the crude product with Soxhlet extractor for $24 \mathrm{~h}$. Brick-red powder was obtained after drying the product in a vacuum oven at $120{ }^{\circ} \mathrm{C}$ for $8 \mathrm{~h}$. Yield: $0.57 \mathrm{~g}$. Elemental anal. C: $81.59 \%$; $: 4.78 \%$; $: 1.14 \%$; $\mathrm{Al}: 0.036 \%$ (the content of Al was measured by ICP analysis).

\subsection{Preparation of NHCP-4}

Dichloromethane $(30 \mathrm{~mL})$ was injected along with TP (1.6 mmol, $0.41 \mathrm{~g}$ ), 4,6-DCM (2.4 mmol, $0.36 \mathrm{~g}$ ) and anhydrous aluminium chloride $(10 \mathrm{mmol}, 1.33 \mathrm{~g})$ in a $100 \mathrm{~mL}$ roundbottom flask under inert atmosphere. The mixture was stirred for $24 \mathrm{~h}$ under $40{ }^{\circ} \mathrm{C}$ to complete the polymerization. After cooling to room temperature, the reaction was quenched with a small amount of methanol. The solution was filtered to collect the residue under extraction filtration. Then, the residue was washed with diluted hydrochloric acid, methanol and dichloromethane, in sequence. For further purification, methanol was used to extract the crude product with Soxhlet extractor for $24 \mathrm{~h}$. Brick-red powder was obtained after drying the product in a vacuum oven at $120{ }^{\circ} \mathrm{C}$ for $8 \mathrm{~h}$. Yield: $0.68 \mathrm{~g}$. Elemental anal. C: $75.81 \%$; $\mathrm{H}: 4.90 \%$; $: 4.54 \%$; Al: $0.03 \%$ (the content of Al was measured by ICP analysis).

\subsection{Characterization}

Infrared spectra ( $\mathrm{KBr}$ pellets) were obtained in the 3600$400 \mathrm{~cm}^{-1}$ range using a Bomem MB-102 IR spectrometer. Solid state ${ }^{13} \mathrm{C}$ cross-polarization magic angle spinning (CP/MAS) NMR spectra were obtained from a Bruker Avance II $400 \mathrm{WB}$ $400 \mathrm{MHz}$ spectrometer equipped with a $4 \mathrm{~mm}$ doubleresonance MAS probe and a spinning frequency of $8 \mathrm{kHz}$. Scanning electron microscope (SEM) experiments were performed on a JSM 6700 at $10.0 \mathrm{kV}$ with gold sputter-coated on the surface of samples. UV-visible (UV-Vis) spectra of the organic compound solutions were recorded using a Shimadzu UV-2600 spectrophotometer. Powder X-ray diffraction data were collected on a Rigaku-Dmax 2500 diffractometer in a $2 \theta$ range of $4-50^{\circ}$ using $\mathrm{Cu} \mathrm{K} \alpha$ radiation. Thermogravimetric analysis (TGA) was performed on a Mettler-Toledo TGA/ SDTA851e thermal analyzer ranging from 20 to $1000{ }^{\circ} \mathrm{C}$ under nitrogen atmosphere. Elemental analysis (EA) was performed on an Elementar Vario MICRO elemental analyzer. Inductively coupled plasma analysis (ICP) was performed on an Ultima-2 ICP emission spectrometer. $\mathrm{N}_{2}$ sorption isotherms were measured using a Micromeritics ASAP 2020 surface area and porosimetry analyser at $77 \mathrm{~K}$. The Brunauer-EmmettTeller (BET) surface areas of the polymers were calculated by the BET equation over a relative pressure $\left(P / P_{0}\right)$ range of $0.01-$ 0.1 . Pore size distributions were derived from $\mathrm{N}_{2}$ adsorption isotherms using the nonlocal density functional theory (NLDFT) model. All samples were degassed under vacuum at $150{ }^{\circ} \mathrm{C}$ for $10 \mathrm{~h}$ before measurements.

\subsection{Adsorption studies}

Four typical organic micropollutants, 2,4-DCP, 2-NO, BPA and $\mathrm{BPS}$, were used to determine the adsorption performance of the NHCPs. In a typical kinetics experiment with 2,4-DCP forNHCP1, $20 \mathrm{mg}$ of NHCP-1 was placed in a $100 \mathrm{~mL}$ round-bottom flask with a stir bar. A few drops of deionized water were added to wet the adsorbent. Then, $20 \mathrm{~mL}$ of the $2,4-\mathrm{DCP}$ solution $(0.1 \mathrm{mM})$ was poured into a flask and stirred at $22{ }^{\circ} \mathrm{C}$. Subsequently, $2 \mathrm{~mL}$ of the suspension was removed by syringe and filtered through a $0.2 \mu \mathrm{m}$ PTFE membrane filter at different intervals $(10 \mathrm{~s}, 20 \mathrm{~s}$, $40 \mathrm{~s}, 1 \mathrm{~min}, 5 \mathrm{~min}, 10 \mathrm{~min}, 20 \mathrm{~min}$ ). UV-Vis spectroscopy was performed to determine the concentration of the pollutant in the solution based on calibration with measured molar extinction coefficients $\left(\varepsilon, \mathrm{M}^{-1} \mathrm{~cm}^{-1}\right)$ for 2,4-DCP (2080 at $\lambda_{\max }=284$ $\mathrm{nm}), 2-\mathrm{NO}\left(4350\right.$ at $\left.\lambda_{\max }=273 \mathrm{~nm}\right), \mathrm{BPA}\left(3250\right.$ at $\left.\lambda_{\max }=276 \mathrm{~nm}\right)$ and BPS (19 010 at $\lambda_{\max }=259 \mathrm{~nm}$ ). In addition, NHCP-3 was selected as the adsorbent to study the thermodynamic adsorption experiments. The initial pollutant concentration varied from $0.05 \mathrm{mM}$ to $0.4 \mathrm{mM}$. First, $1 \mathrm{mg}$ of NHCP-3 was introduced into a $20 \mathrm{~mL}$ vial with $10 \mathrm{~mL}$ of the pollutant solution at a certain concentration. Then, the suspension was stirred for $6 \mathrm{~h}$ at $22{ }^{\circ} \mathrm{C}$ to reach equilibrium. Then, $2 \mathrm{~mL}$ of the suspension was taken by syringe and filtered through a $0.2 \mu \mathrm{m}$ PTFE membrane filter. The filtrate was measured by UV-Vis spectroscopy. The adsorption isotherms were fitted by the Langmuir isotherm model ${ }^{29}$ and the Freundlich isotherm model. ${ }^{30}$

The Langmuir isotherm model can be represented as follows:

$$
\frac{1}{q_{\mathrm{e}}}=\frac{1}{q_{\mathrm{m}}}+\frac{1}{q_{\mathrm{m}} K_{\mathrm{L}} c_{\mathrm{e}}}
$$

The Freundlich isotherm model can be represented as follows:

$$
\ln q_{\mathrm{e}}=\ln K_{\mathrm{F}}+\frac{1}{n} \ln c_{\mathrm{e}}
$$

where $q_{\mathrm{e}}\left(\mathrm{mg} \mathrm{g}^{-1}\right)$ is the adsorption capacity of the pollutant, $q_{\mathrm{m}}$ $\left(\mathrm{mg} \mathrm{g}^{-1}\right)$ is the maximum adsorption capacity of the pollutant at equilibrium, $c_{\mathrm{e}}\left(\mathrm{mg} \mathrm{L}^{-1}\right)$ is the residual pollutant concentration at equilibrium, $K_{\mathrm{L}}$ is the Langmuir constant, $K_{\mathrm{F}}$ and $n$ are the Freundlich constants related to adsorption capacity and adsorption intensity, respectively. 


\subsection{NHCPs regeneration}

Initially, $20 \mathrm{mg}$ of the NHCP was wetted with a few drops of deionized water in a $100 \mathrm{~mL}$ round-bottom flask under ultrasonication. A stock solution of BPA $(20 \mathrm{~mL}, 0.1 \mathrm{mM})$ was added. The mixture was then stirred for $30 \mathrm{~min}$ at $22{ }^{\circ} \mathrm{C}$ and centrifuged for $10 \mathrm{~min}$ at speed of $8000 \mathrm{rpm}$. The residual concentration of the pollutant in the filtrate was determined by UV-Vis spectroscopy. The regeneration of the adsorbent was achieved by soaking the adsorbed NHCP in ethanol $(10 \mathrm{~mL} \times 3)$ for 10 min under ultrasonication. The fresh adsorbent was recovered by centrifugation and dried under vacuum at $120^{\circ} \mathrm{C}$. Then, the adsorption performance of the NHCP was tested four more times to evaluate recyclability.

\subsection{Fast flow tests}

Initially, $2 \mathrm{mg}$ of the adsorbent was placed on the bottom of a syringe equipped with a $0.2 \mu \mathrm{m}$ PTFE membrane filter. Then, $2 \mathrm{~mL}$ of deionized water was added into the syringe to soak the adsorbent. The syringe was pushed to remove water and make the adsorbent form a thin layer on the membrane filter. Subsequently, $2 \mathrm{~mL}$ of the pollutant stock solution was added and pushed through the adsorbent within $5 \mathrm{~s}$ (flow rate of 24 $\left.\mathrm{mL} \min ^{-1}\right)$. The filtrate was then collected and measured by UVVis spectroscopy to calculate the efficiency of the pollutant removal.

\section{Result and discussion}

The N-containing hyper-crosslinked porous polymers can be easily obtained under mild reaction conditions by a FriedelCrafts alkylation reaction. The detailed synthesis routes are shown in Fig. 1d. The structural information of these polymers can be characterized by their FT-IR spectra (Fig. 2a and S1 $\dagger$ ). The emergence of strong peaks at 1600 and $1687 \mathrm{~cm}^{-1}$ can be ascribed to the stretching vibration adsorption of aromatic conjugated structures, which indicates the successful
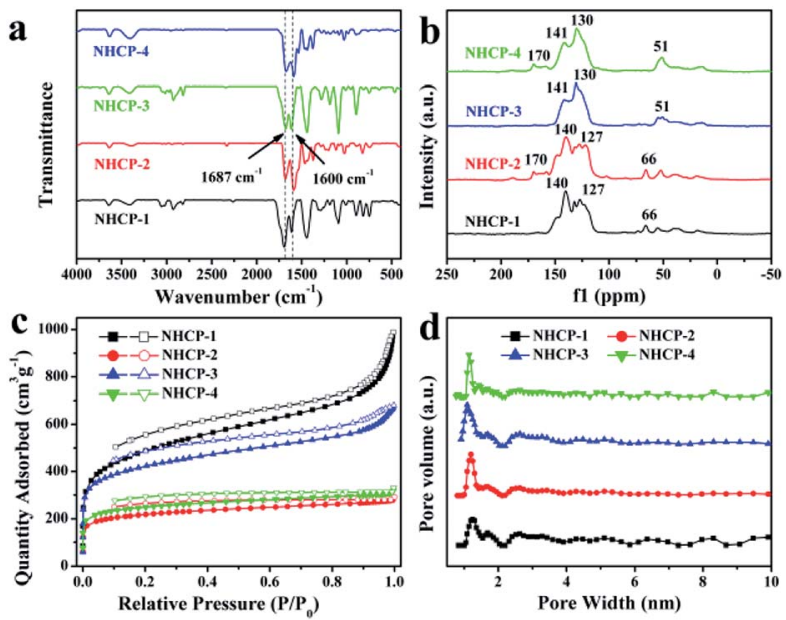

Fig. 2 (a) FT-IR spectra of NHCPs. (b) Solid-state ${ }^{13} \mathrm{C}$ CP/MAS NMR spectra of NHCPs. (c) $\mathrm{N}_{2}$ sorption isotherms of NHCPs measured at 77 K. (d) Pore size distributions of NHCPs. polymerization between monomers and crosslinkers. The disappearance of the $\mathrm{C}-\mathrm{Br}$ stretching band at $997 \mathrm{~cm}^{-1}$ in NHCP-1 and NHCP-3 also reveals the generation of covalent bonds between the benzene and pyrazine rings. The same explanation applies equally well to the absence of peaks at $809 \mathrm{~cm}^{-1}$ in the FTIR spectra of NHCP-2 and NHCP-4.

Solid state ${ }^{13} \mathrm{C}$ NMR spectra were measured to further investigate the structures of the NHCPs, as shown in Fig. 2b. In the solid state ${ }^{13} \mathrm{C}$ NMR spectra of NHCP-1 and NHCP-2, the peaks at around 127-140 ppm are ascribed to the various carbon atoms of the aromatic structural units and the peak at $66 \mathrm{ppm}$ is assigned to the quaternary carbon atoms of the spirobifluorene units. ${ }^{31}$ Moreover, in the spectra of NHCP-3 and NHCP-4, the signals derived from the aromatic structural units are observed at around 130-140 ppm, while the signal at $51 \mathrm{ppm}$ corresponds to the tertiary carbon atoms of the triptycene units. $^{32}$ Furthermore, the evident peaks at around $170 \mathrm{ppm}$ observed in the spectra of NHCP-2 and NHCP-4 can be assigned to the carbon atoms adjacent to nitrogen atoms in pyrimidine rings. However, similar signals of the carbon atoms in pyrazine rings are not evidently observed in the solid state spectra of NHCP-1 and NHCP-3, probably because of the low levels of the pyrazine structures.

Residual content of aluminium in the NHCPs indicates almost complete removal of the catalysts. These polymers showed high thermostability and remain undecomposed even at $300{ }^{\circ} \mathrm{C}$ under nitrogen atmosphere (Fig. S2 $\dagger$ ). All of them are present as amorphous powders, which was verified by the powder X-ray diffraction data, as seen in Fig. S3. $\uparrow$ The particle size and morphology of these polymers can be observed by scanning electron microscopy (SEM) analysis (Fig. S4†). NHCP-1 exhibits an irregularly shaped morphology, while NHCP-3 has spherical particle morphology with a relatively uniform diameter of $500 \mathrm{~nm}$. The pyrimidine-crosslinked polymers NHCP-2 and NHCP-4 reveal similar spherical particles with a particle size of around $2 \mu \mathrm{m}$.

To further investigate the porosity of the NHCPs, nitrogen sorption isotherms were measured at $77 \mathrm{~K}$, as shown in Fig. 2c. All the NHCPs exhibit classical type I isotherms with a steep rise at lower relative pressures $\left(P / P_{0}<0.01\right)$, suggesting the presence of permanent micropores. In addition, NHCP-2 and NHCP-4 show type IV isotherms with adsorption hysteresis at higher relative pressures, indicating the coexistence of mesopores and even small macropores. The BET model was used to calculate the surface areas of these polymers. The detailed porosity data of the NHCPs is summarized in Table 1. The resulting BET surface areas are $1768,810,1544$ and $930 \mathrm{~m}^{2} \mathrm{~g}^{-1}$ for NHCP-1, NHCP-2, NHCP-3 and NHCP-4, respectively. The micropore volumes were calculated by the $t$-plot method, and NHCP-3 was found to have the highest micropore volume of $0.27 \mathrm{~cm}^{3} \mathrm{~g}^{-1}$. The total pore volumes were estimated from nitrogen isotherms at $P / P_{0}=0.99$, with the highest value of $1.53 \mathrm{~cm}^{3} \mathrm{~g}^{-1}$ for NHCP1. As shown in Table 1, pyrazine-based NHCP-1 and NHCP-3 perform better than pyrimidine-based NHCP-2 and NHCP-4 in porosity. The adsorption performance of SBF-containing NHCP2 is not as good as that of TP-containing NHCP-4, while SBFcontaining NHCP-1 has higher surface area than TP- 
Table 1 Porosity data for the NHCPs

\begin{tabular}{lclll}
\hline Polymers & $S_{\text {BET }}\left(\mathrm{m}^{2} \mathrm{~g}^{-1}\right)$ & $V_{\text {Micro }}{ }^{a}\left(\mathrm{~cm}^{3} \mathrm{~g}^{-1}\right)$ & $V_{\text {Total }}^{b}\left(\mathrm{~cm}^{3} \mathrm{~g}^{-1}\right)$ & 1.53 \\
NHCP-1 & & 0.19 & 0.45 & 0.12 \\
NHCP-2 & 1768 & 0.17 & 1.05 & 0.38 \\
NHCP-3 & 810 & 0.27 & 0.51 & 0.26 \\
NHCP-4 & 1544 & 0.21 & 0.41
\end{tabular}

${ }^{a}$ Micropore volumes were calculated by $t$-plot method. ${ }^{b}$ Pore volumes were calculated from nitrogen isotherms at $P / P_{0}=0.99$ and $77 \mathrm{~K}$.

containing NHCP-3. The ratios of the micropore volume to total pore volume range from 0.12 to 0.41 . The pore size distributions (PSD) were calculated using the nonlocal density functional theory (NLDFT). As shown in Fig. 2d, all the polymers show a similar tendency with a narrow distribution of micropores centered at $1.2 \mathrm{~nm}$ and a broad distribution of mesopores centered at $2.5 \mathrm{~nm}$, which are in good agreement with the shapes of the corresponding nitrogen isotherms.

Considering the excellent porosity structure and high stability of the NHCPs, we further tested the performance of these polymers towards the adsorption removal of organic micropollutants from water solutions. A series of contaminants with different sizes were chosen as experimental subjects, namely, 2,4-DCP, 2-NO, BPA and BPS. PTFE membrane filters $(0.2 \mu \mathrm{m})$ were selected to filter the solution in view of the balance of adsorption effect and consumption cost (Fig. S5 $\dagger$ ). The time-dependent adsorption removal efficiency of different pollutants is revealed in Fig. 3 and S6-S9. $\dagger$ As shown in Fig. 3, with the initial concentrations of $0.1 \mathrm{mM}$, all the polymers can efficiently remove $90 \%$ of the pollutants in the first 10 seconds and almost achieve adsorption equilibrium after 1 minute. Particularly, NHCP-3 can remove nearly all the contaminants within 10 seconds. After 5 minutes, the removal efficiency of NHCP-3 for 2,4-DCP, 2-NO, BPA and BPS reached 99.0, 99.5, 99.4 and $99.9 \%$, respectively (Fig. 4). The pollutant removal performance of NHCP-3 is better than that of other NHCPs.

The polymer NHCP-3 shows excellent adsorption performance towards organic micropollutants, which may benefit from its pore structure and pore size distributions. First, NHCP3 has a high surface area (up to $1544 \mathrm{~m}^{2} \mathrm{~g}^{-1}$ ) and high micropore volume (up to $0.27 \mathrm{~cm}^{3} \mathrm{~g}^{-1}$ ). The abundant micropores provide sufficient active sites to adsorb organic molecules. Second, NHCP-3 has the right amount of mesopores, which can supply the necessary pathways to the micropores since bulky organic molecules need to go through mesopores to approach micropores. The pore size distribution of the mesopores affects the diffusion rate of these molecules. Hence, the appropriate pore size distribution is helpful to the diffusion of solutes in pores with enhanced adsorption capacity and efficiency. Both pore structures and pore size distributions contribute to the excellent adsorption performance.

The adsorption process for organic molecules was realized by physical adsorption interactions, including van der Waals forces and hydrogen bonding. The physical adsorption is usually classified into Langmuir monolayer adsorption, BET multilayer adsorption and so on. The Langmuir isotherm model and the Freundlich isotherm model are generally used to fit the adsorption isotherm to determine the specific type of adsorption process. To further understand the thermodynamic adsorption process, NHCP-3 was selected to perform adsorption experiments with pollutant solutions of different initial concentrations. Then, the adsorption results were fitted by the two isotherm models (Fig. 5, S10 and Table S1 $\dagger$ ). From the fitting results, the correlation coefficients of Langmuir model $\left(R_{\mathrm{L}}^{2}\right)$ were 0.991, 0.996, 0.979 and 0.961 for 2,4-DCP, 2NO, BPA and BPS, respectively, while the corresponding correlation coefficients for the Freundlich model $\left(R_{\mathrm{F}}{ }^{2}\right)$ were $0.910,0.850,0.819$ and 0.879 . The values of $R_{\mathrm{L}}{ }^{2}$ are much
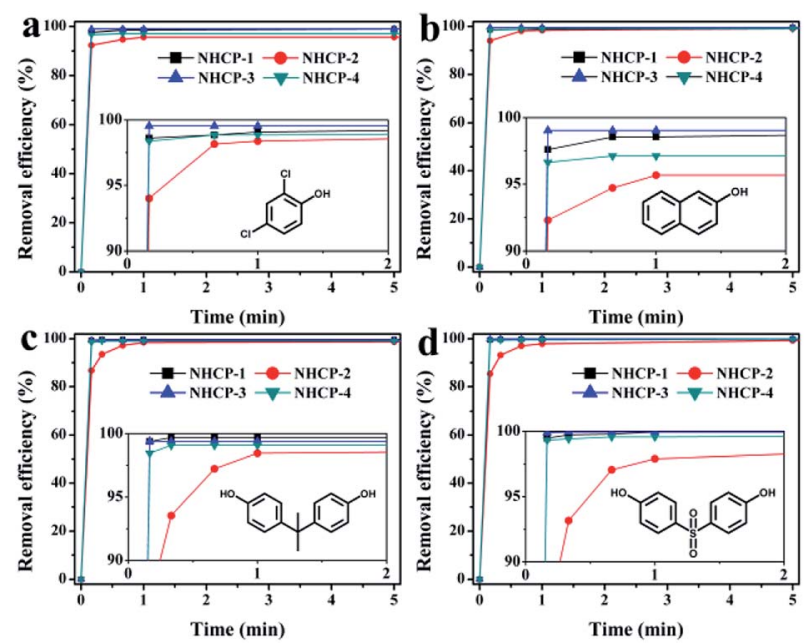

Fig. 3 Time-dependent adsorption removal efficiency of 2,4-DCP (a), 2-NO (b), BPA (c) and BPS (d) solution ( $0.1 \mathrm{mM}$ ) by $1 \mathrm{mg} \mathrm{mL}^{-1}$ sample of the NHCP. Insets display partially enlarged details.
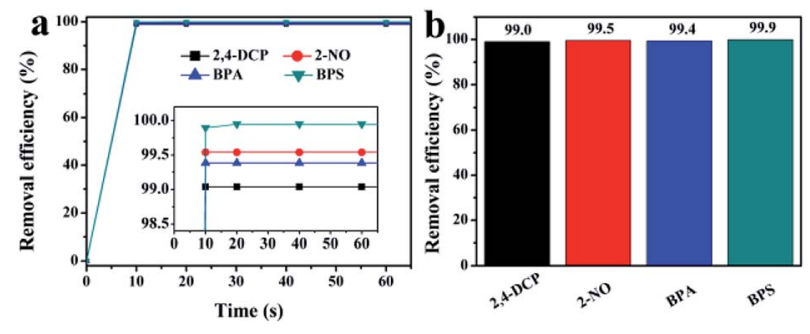

Fig. 4 (a) Adsorption removal efficiency of the pollutant solution (0.1 $\mathrm{mM}$ ) by $1 \mathrm{mg} \mathrm{mL}^{-1} \mathrm{NHCP}-3$. (b) Percentage removal efficiency for the pollutants solution ( $0.1 \mathrm{mM}$ ) by $1 \mathrm{mg} \mathrm{mL}^{-1} \mathrm{NHCP}-3$, determined after 5 minutes. Insets display partially enlarged details. 

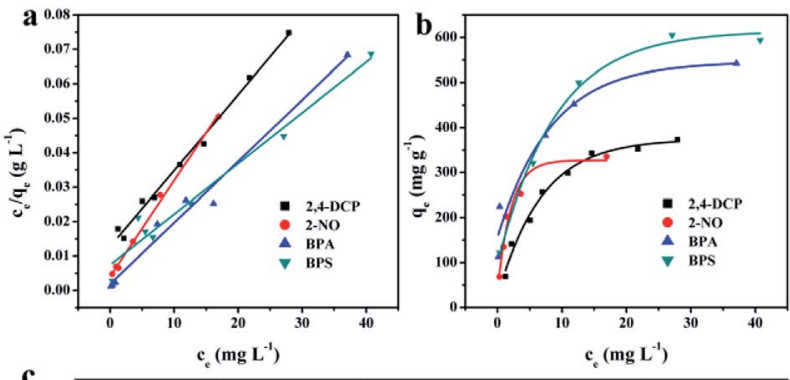

\begin{tabular}{|c|c|c|c|c|}
\hline Pollutants & $2,4-\mathrm{DCP}$ & 2-NO & BPA & BPS \\
\hline $\mathbf{q}_{\mathbf{m}}\left(\mathbf{m g ~ g}^{-1}\right)$ & 455 & 358 & 562 & 680 \\
\hline $\mathbf{R}_{\mathbf{L}}{ }^{2}$ & 0.991 & 0.996 & 0.979 & 0.961 \\
\hline
\end{tabular}

Fig. 5 (a) The Langmuir isotherm model and (b) the adsorption isotherms for 2,4-DCP, 2-NO, BPA, and BPS; (c) the maximum uptake capacities $\left(q_{m}\right)$ for 2,4-DCP, 2-NO, BPA and BPS by NHCP-3. The amount of the adsorbent used in this study is $0.1 \mathrm{mg} \mathrm{mL}^{-1}$.

higher than those of $R_{\mathrm{F}}^{2}$, indicating that the adsorption process for the organic pollutants is close to the Langmuir monolayer adsorption model. This proves that the inner surface of the adsorbents is covered by a single layer of organic molecules, which is consistent with the conclusion of previous report. ${ }^{25}$ Using the Langmuir adsorption model, the maximum adsorption capacities $\left(q_{\mathrm{m}}\right)$ of NHCP-3 were calculated to be $455,358,562$ and $680 \mathrm{mg} \mathrm{g}^{-1}$ for 2,4-DCP, 2-NO, BPA and BPS, respectively. These values are much higher than those of the adsorbents reported previously. ${ }^{21,26,33-35}$ The maximum adsorption capacities of BPA, as an example, are summarized in Table 2.

To test the recyclability of the adsorbents, NHCP-3 was selected to perform adsorption and regeneration experiments with BPA (Fig. 6a). BPA can be easily removed from NHCP-3 by immersion in ethanol at room temperature. After five consecutive recycling experiments, the BPA removal efficiency remained at $99.1 \%$ with almost no loss. In order to simulate flowing wastewater at high speed, we also performed fast flow experiments. The practical adsorption performance of NHCP-3 and the activated carbons (ACs) were tested in the case of fast flow at the speed of $24 \mathrm{~mL} \mathrm{~min}^{-1}$. The results are shown in Fig. 6b. NHCP-3 exhibits removal efficiency of $96.8 \%, 99.5 \%, 98.2 \%$ and $86.5 \%$ for 2,4-DCP, 2-NO, BPA and BPS, respectively, while AC correspondingly displays removal efficiency of $6.5 \%, 2.2 \%, 1.8 \%$ and $5.3 \%$. These results indicate that the NHCPs are efficient and practical adsorbents for use in wastewater treatment.

Table 2 Comparison of adsorption capacities of BPA with other adsorbents

\begin{tabular}{lcl}
\hline Adsorbents & $\begin{array}{l}\text { Adsorption capacity } \\
\left(\mathrm{mg} \mathrm{g}^{-1}\right)\end{array}$ & Ref. \\
\hline EPI-CDP & 84 & 33 \\
P-CDP & 88 & 21 \\
Graphene & 182 & 34 \\
Fe/OMC & 311 & 35 \\
CalP4 & 403 & 26 \\
NHCP-3 & 562 & This work
\end{tabular}

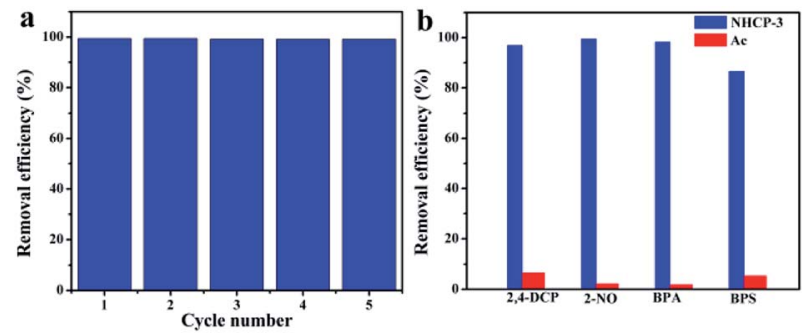

Fig. 6 (a) Reusability of the NHCP-3 for BPA in 30 minutes. (b) Removal efficiency of 2,4-DCP (0.1 mM), 2-NO (0.1 mM), BPA (0.1 mM) and BPS $(0.1 \mathrm{mM})$ obtained by rapidly flowing the pollutants solution through NHCP-3 (blue) and Ac (red), respectively. The amount of the adsorbent used in this study is $1 \mathrm{mg} \mathrm{mL}^{-1}$.

\section{Conclusions}

In summary, we have synthesized four twisted molecule-based hierarchically hyper-crosslinked porous polymers with high surface areas and rich porous structures using a simple synthesis method. The NHCPs exhibit excellent adsorption performance towards pollutants in aqueous solution with rapid and high adsorption capacities. In addition, the polymers can be easily regenerated at least five times without any loss in efficiency. Fast flow experiments were also performed to simulate practical flowing wastewater at a high speed, revealing the efficient removal performance of the polymers in seconds. The experiments reveal that the NHCPs possess characteristics of facile synthesis, high removal efficiency and high adsorption capacity, which make them potential adsorbents for application in wastewater treatment.

\section{Conflicts of interest}

There are no conflicts to declare.

\section{Acknowledgements}

This study was supported by the National Key R\&D Program of China (2016YFB0600903), Key Research Program of Frontier Sciences, CAS (QYZDB-SSW-SLH019) and National Nature Science Foundation of China (21771177, 51603206 and 21603229).

\section{References}

1 R. P. Schwarzenbach, B. I. Escher, K. Fenner, T. B. Hofstetter, C. A. Johnson, U. von Gunten and B. Wehrli, Science, 2006, 313, 1072-1077.

2 M. A. Montgomery and M. Elimelech, Environ. Sci. Technol., 2007, 41, 17-24.

3 S. D. Richardson and S. Y. Kimura, Anal. Chem., 2016, 88, 546-582.

4 Y. Luo, W. Guo, H. H. Ngo, L. D. Nghiem, F. I. Hai, J. Zhang, S. Liang and X. C. Wang, Sci. Total Environ., 2014, 473-474, 619-641. 
5 B. Petrie, R. Barden and B. Kasprzyk-Hordern, Water Res., 2015, 72, 3-27.

6 O. Rozas, C. Vidal, C. Baeza, W. F. Jardim, A. Rossner and H. D. Mansilla, Water Res., 2016, 98, 109-118.

7 M. A. Shannon, P. W. Bohn, M. Elimelech, J. G. Georgiadis, B. J. Marinas and A. M. Mayes, Nature, 2008, 452, 301-310.

8 R. Rosal, A. Rodriguez, J. A. Perdigon-Melon, A. Petre, E. Garcia-Calvo, M. J. Gomez, A. Aguera and A. R. Fernandez-Alba, Water Res., 2010, 44, 578-588.

9 I. Oller, S. Malato and J. A. Sanchez-Perez, Sci. Total Environ., 2011, 409, 4141-4166.

10 B. P. Tripathi, N. C. Dubey and M. Stamm, J. Hazard. Mater., 2013, 252-253, 401-412.

11 A. K. Gautam, C. Lai, H. Fong and T. J. Menkhaus, J. Membr. Sci., 2014, 466, 142-150.

12 A. Dabrowski, P. Podkoscielny, Z. Hubicki and M. Barczak, Chemosphere, 2005, 58, 1049-1070.

13 A. Zhou, X. Ma and C. Song, J. Phys. Chem. B, 2006, 110, 4699-4707.

14 C. Yin, M. Aroua and W. Daud, Sep. Purif. Technol., 2007, 52, 403-415.

15 E. Deliyanni, M. Seredych and T. J. Bandosz, Langmuir, 2009, 25, 9302-9312.

16 H. L. Jiang and Q. Xu, Chem. Commun., 2011, 47, 3351-3370.

17 Z. Hasan, J. Jeon and S. H. Jhung, J. Hazard. Mater., 2012, 209-210, 151-157.

18 N. A. Khan, Z. Hasan and S. H. Jhung, J. Hazard. Mater., 2013, 244-245, 444-456.

19 C. Chen, D. Chen, S. Xie, H. Quan, X. Luo and L. Guo, ACS Appl. Mater. Interfaces, 2017, 9, 41043-41054.
20 D. Chen, C. Chen, W. Shen, H. Quan, S. Chen, S. Xie, X. Luo and L. Guo, Adv. Powder Technol., 2017, 28, 1769-1779.

21 A. Alsbaiee, B. J. Smith, L. Xiao, Y. Ling, D. E. Helbling and W. R. Dichtel, Nature, 2016, 529, 190-194.

22 S. Lan, S. Zhan, J. Ding, J. Ma and D. Ma, J. Mater. Chem. A, 2017, 5, 2514-2518.

23 F. Zhao, E. Repo, D. Yin, L. Chen, S. Kalliola, J. Tang, E. Iakovleva, K. C. Tam and M. Sillanpaa, Sci. Rep., 2017, 7, 15811.

24 Y. Liu and K. Landskron, J. Mater. Chem. A, 2017, 5, 2352323529.

25 B. Shi, H. Guan, L. Shangguan, H. Wang, D. Xia, X. Kong and F. Huang, J. Mater. Chem. A, 2017, 5, 24217-24222.

26 D. Shetty, I. Jahovic, J. Raya, Z. Asfari, J. C. Olsen and A. Trabolsi, ACS Appl. Mater. Interfaces, 2018, 10, 2976-2981.

27 Z. Jia, J. Pan and D. Yuan, ChemistryOpen, 2017, 6, 554-561.

28 D. Chen, S. Gu, Y. Fu, X. Fu, Y. Zhang, G. Yu and C. Pan, New J. Chem., 2017, 41, 6834-6839.

29 H. Liu, X. Cai, Y. Wang and J. Chen, Water Res., 2011, 45, 3499-3511.

30 I. X. Garcia-Zubiri, G. Gonzalez-Gaitano and J. R. Isasi, J. Colloid Interface Sci., 2009, 337, 11-18.

31 S. Gu, J. Guo, Q. Huang, J. He, Y. Fu, G. Kuang, C. Pan and G. Yu, Macromolecules, 2017, 50, 8512-8520.

32 C. Zhang, P.-C. Zhu, L. Tan, J.-M. Liu, B. Tan, X.-L. Yang and H.-B. Xu, Macromolecules, 2015, 48, 8509-8514.

33 N. Morin-Crini and G. Crini, Prog. Polym. Sci., 2013, 38, 344368.

34 J. Xu, L. Wang and Y. Zhu, Langmuir, 2012, 28, 8418-8425.

35 L. Tang, Z. Xie, G. Zeng, H. Dong, C. Fan, Y. Zhou, J. Wang, Y. Deng, J. Wang and X. Wei, RSC Adv. , 2016, 6, 25724-25732. 